\title{
Characteristic cardiac phenotypes are detected by cardiovascular magnetic resonance in patients with different clinical phenotypes and genotypes of mitochondrial myopathy
}

Anca Florian ${ }^{1}$, Anna Ludwig², Bianca Stubbe-Dräger ${ }^{3}$, Matthias Boentert $^{3}$, Peter Young ${ }^{3}$, Johannes Waltenberger ${ }^{1}$, Sabine Rösch², Udo Sechtem² and Ali Yilmaz ${ }^{1 *}$

\begin{abstract}
Background: Mitochondrial myopathies (MM) are a heterogeneous group of inherited conditions resulting from a primary defect in the mitochondrial respiratory chain with consecutively impaired cellular energy metabolism. Small sized studies using mainly electrocardiography (ECG) and echocardiography have revealed cardiac abnormalities ranging from conduction abnormalities and arrhythmias to hypertrophic or dilated cardiomyopathy in these patients. Recently, characteristic patterns of cardiac involvement were documented by cardiovascular magnetic resonance (CMR) in patients with chronic progressive external ophthalmoplegia (CPEO)/Kearns-Sayre syndrome (KSS) and with mitochondrial encephalopathy with lactic acidosis and strokelike episodes (MELAS). The present study aimed to characterize the prevalence and pattern of cardiac abnormalities and to test the additional diagnostic value of CMR in this patient population. The hypothesis that different neuromuscular MM syndromes present with different cardiac disease phenotypes was evaluated.
\end{abstract}

Methods: Sixty-four MM patients ( $50 \pm 15$ years, $44 \%$ male) and 25 matched controls ( $52 \pm 14$ years, $36 \%$ male) prospectively underwent cardiac evaluations including CMR (comprising cine- and late-gadolinium-enhancement (LGE) imaging). Based on the neuromuscular phenotype and genotype, the patients were grouped: a) CPEO/KSS ( $N=33)$; $b$ ) MELAS/-like ( $N=11)$; c) myoclonic eppilepsy with ragged-red fibers (MERRF) ( $N=3)$ and d) other non-specific MM forms $(\mathrm{N}=17)$.

(Continued on next page)

\footnotetext{
* Correspondence: ali.yilmaz@ukmuenster.de

${ }^{1}$ Department of Cardiology and Angiology, University Hospital Münster,

Albert-Schweitzer-Campus 1, building A1, 48149 Münster, Germany

Full list of author information is available at the end of the article
} 
(Continued from previous page)

Results: Among the 64 MM patients, 34 (53\%) had at least one abnormal CMR finding: 18 (28 \%) demonstrated an impaired left ventricular ejection-fraction (LV-EF <60 \%), 14 (22\%) had unexplained LV hypertrophy and 21 (33 \%) were LGE-positive. Compared to controls, MM patients showed significantly higher maximal wall thickness (10 \pm 3 vs. $8 \pm 2 \mathrm{~mm}, \mathrm{p}=0.005$ ) and concentricity (LV mass to end-diastolic volume: $0.84 \pm 0.27$ vs. $0.67 \pm 0.11, p<0.0001$ ) with frequent presence of non-ischemic LGE (30 \% vs. $0 \%, p=0.001)$. CPEO/KSS showed a predominantly intramural pattern of LGE mostly confined to the basal LV inferolateral wall (8/10; $80 \%)$ in addition to a tendency toward concentric remodelling. MELAS/-like patients showed the highest frequency of cardiac disease (in 10/11 (91\%)), a mostly concentric LV hypertrophy $(6 / 9 ; 67 \%)$ with or without LV systolic dysfunction and a predominantly focal, patchy LGE equally distributed among LV segments (8/11; 73 \%). Patients with MERRF and non-specific MM had no particular findings. Pathological CMR findings indicating cardiac involvement were detected significantly more often than pathological ECG results or elevated cardiac serum biomarkers (34 (53 \%) vs. 18 (28 \%) vs. 21 (33 \%); p=0.008).

Conclusion: Cardiac involvement is a frequent finding in MM patients - and particularly present in KSS/CPEO as well as MELAS/-like patients. Despite a high variability in clinical presentation, CPEO/KSS patients typically show an intramural pattern of LGE in the basal inferolateral wall whereas MELAS patients are characterized by overt concentric hypertrophy and a rather unique, focally accentuated and diffusely distributed LGE.

Keywords: Mitochondrial myopathy, Cardiomyopathy, Cardiovascular magnetic resonance, MELAS, CPEO

\section{Background}

Mitochondrial myopathies (MM) are a heterogeneous group of inherited conditions resulting from a primary defect in the mitochondrial respiratory chain with consecutively impaired cellular energy metabolism affecting multiple organ systems. Despite the high variability in clinical presentation and the poor genotype-phenotype correlation, several syndromes with characteristic symptoms have been defined [1]. These disorders have a progressive course associated with different degrees of neurological disability and in some instances with premature death primarily due to cardiac and neurological adverse events [2-5].

The overall prevalence of MM-related cardiomyopathy is difficult to estimate and varies according to syndrome and to the diagnostic approach used $[6,7]$. Cardiac abnormalities ranging from preexcitation, conduction blocks and arrhythmias to dilated or hypertrophic cardiomyopathy phenotypes have been described in several syndromes like chronic progressive external ophthalmoplegia (CPEO), Kearns-Sayre syndrome (KSS), mitochondrial encephalopathy with lactic acidosis and stroke-like episodes (MELAS), $\underline{\text { myoclonic }}$ epilepsy with ragged-red fibers (MERRF) and Leigh syndrome. These $\overline{\text { data derive }}$ from case reports and small sized studies using mainly electrocardiography (ECG) and echocardiography for cardiac evaluation [2, 4, 5, 8-12].

Cardiovascular magnetic resonance (CMR) is a highly sensitive tool for depicting myocardial abnormalities in MM patients including tissue damage by late gadolinium enhancement (LGE) [13-16]. Recently, two small sized CMR studies suggested that characteristic patterns of cardiac involvement might be present in some of the above syndromes $[17,18]$. As no disease modifying therapy exists, an early diagnosis of cardiac involvement - particularly by CMR studies - would permit a timely initiation of appropriate treatment strategies with potential improvement in patient prognosis [19].

The present study aimed to characterize the prevalence and pattern of cardiac abnormalities in a group of MM patients with different clinical phenotypes and to test the additional diagnostic value of CMR in this patient population. Moreover, the hypothesis that different neuromuscular MM syndromes present with different cardiac disease phenotypes was evaluated.

\section{Methods}

\section{Study population}

Sixty-four patients with known MM were prospectively enrolled between 2009 and 2014 as participants of the "Mito-HERZ" study that was already described elsewhere [18]. All patients underwent cardiac and neurological evaluations including multi-parametric CMR. The clinical diagnosis of MM had been previously confirmed based on molecular genetic testing and/or skeletal biopsy with appropriate findings in all patients [10]. Exclusion criteria were presence of claustrophobia and contraindications to CMR or to gadolinium contrast administration.

Based on the clinical (neuromuscular) phenotype and genetic findings, the patients were further grouped as follows: (1) CPEO/KSS ( $\mathrm{N}=33 ; 29$ with $\mathrm{CPEO}$ and 4 with KSS); (2) MELAS and MELAS-like ( $\mathrm{N}=11 ; 7$ with MELAS and 4 with MELAS-like); (3) MERRF $(\mathrm{N}=3)$ and (4) other non-specific MM forms $(\mathrm{N}=17)$ (see also Additional file 1). In addition, 25 healthy individuals matched for age, gender and cardiovascular risk factors with no history of cardiac disease were enrolled between March 2011 and May 2014 and represented the control 
group. Approval of the study protocol was obtained from the local ethics committee, and all participating patients provided written informed consent.

\section{Cardiac evaluation}

Both MM patients and controls underwent cardiac workup including a thorough clinical history, physical examination, 12-lead ECG and CMR. An ECG was considered abnormal whenever at least one of the following findings was present: (1) arrhythmia; (2) conduction abnormalities; (3) isolated ST-segment depression in two or more contiguous leads; (4) isolated inverted T-waves; (5) pathologic Q-waves; (6) a Sokolow-Lyon index $>35 \mathrm{~mm}$ as sign of LV hypertrophy. In the MM patients, blood samples were taken for laboratory analysis including total creatine kinase $(\mathrm{CK})$ and the cardiac biomarkers troponin $\mathrm{T}(\mathrm{TnT})$ and brain natriuretic-peptide (NT-proBNP).

\section{CMR imaging protocol}

ECG-gated CMR studies were performed on 1.5-T scanners (Aera, Siemens Medical Solutions, Erlangen, Germany and Achieva, Philips, Best, The Netherlands) using commercially available cardiac software, electrocardiographic triggering, and cardiac-dedicated surface coils. Cine-imaging was performed using a steady-statefree-precession (SSFP) sequence in three long-axis slices (four-, three- and two-chamber) and a stack of shortaxis slices completely covering the LV. LGE-imaging was performed using a T1-weighted inversion recovery gradient-echo sequence 10-15 $\mathrm{min}$ after intravenous contrast administration $\left(0.15 \mathrm{mmol} / \mathrm{kg}\right.$ Magnevist $\left.{ }^{\circ}\right)$ in the same imaging planes as the cine-images.

\section{CMR image analysis}

CMR analysis was performed off-line by two experienced readers. Ventricular volumes, ejection fraction and LV mass were derived by contouring endo- and epicardial borders on the short-axis cine images and indexed to body surface area. The papillary muscles were included in the LV cavity. LV hypertrophy was considered present whenever maximal end diastolic wall thickness was $\geq 13 \mathrm{~mm}$ in men and $\geq 12 \mathrm{~mm}$ in women. The ratio of LV mass to end-diastolic volume was used as an index of concentric hypertrophy [20]. LGE presence and pattern were visually assessed on the short-and long-axis images by using the AHA 17-segment model. LGE pattern was globally assessed as: ischemic (subendocardial and/or transmural) and non-ischemic (subepicardial and/or intramural). An abnormal CMR study was defined by: (1) a LV ejection fraction (EF) less than $60 \%$ and/or (2) the presence of unexplained LV hypertrophy and/or (3) LGE presence in at least one myocardial segment.

\section{Statistical analysis}

Continuous variables with normal distribution are expressed as mean $\pm \mathrm{SD}$. Categorical variables are expressed as frequency with percentage. Student's t-test was used for comparison of normally distributed characteristics between MM patients and controls. Levene's test was used for testing equality of variances. One-way ANOVA with Bonferroni post hoc correction was used for subgroup multiple comparison analyses. Dunnett's post hoc test was used in the case of inequality of variances. The chi-square test with Yate's correction was used to compare noncontinuous variables expressed as proportions. Statistical analysis was performed using SPSS software for Windows (Version 19.0, IBM Corp., Armonk., NY). A p-value $\leq 0.05$ was considered statistically significant.

\section{Results}

\section{Patient characteristics}

Demographic an main clinical characteristics of both patients and controls are shown in Table 1. Mean age was $50 \pm 15$ years for MM patients and $47 \pm 12$ years for controls. With regard to age, there was no significant difference between MM subgroups $(\mathrm{p}=0.46)$.

Twenty-six percent $(\mathrm{N}=17)$ of the patients had a positive family history for MM. MELAS/-like patients presented significantly more often with a positive family history compared to CPEO/KSS and other MM patients. Thirty-one percent of MM patients $(\mathrm{N}=20)$ had previously received a cardiac diagnosis as follows: arrhythmia $(\mathrm{N}=8)$, impaired LV systolic function $(\mathrm{N}=5)$, LV hypertrophy $(\mathrm{N}=3)$, coronary artery disease (other than infarct $)(\mathrm{N}=3)$ and left bundle branch block $(\mathrm{N}=1)$. Although MELAS/-like patients had an almost double rate of previous heart disease diagnosis compared to the other MM subgroups, this difference was not statistically significant.

Table 2 summarizes the frequencies of different clinical features in $M M$ patients. Nineteen percent of patients $(\mathrm{N}=12)$ presented with chest pain symptoms and $47 \%(\mathrm{~N}=30)$ with exertional dyspnoea. Altogether, $48 \%$ of MM patients had symptoms of possible cardiac origin at inclusion. No significant difference between MM groups regarding cardiac symptoms frequency was noted ( $\mathrm{p}=0.06$ for any possible cardiac symptoms). Interestingly, MELAS/-like patients were the least symptomatic subgroup.

\section{Laboratory results}

As shown in Table $1,13 \%(\mathrm{~N}=8)$ of the MM patients showed an elevated TnT and $25 \%(\mathrm{~N}=16)$ elevated natriuretic peptides at inclusion. Among the $11 \mathrm{CPEO} /$ KSS patients with elevated biomarkers, 10 had elevated NT-proBNP and 1 elevated TnT levels. Six MELAS/-like patients had an increase in biomarkers, TnT elevation 
Table 1 Patient general characteristics

\begin{tabular}{|c|c|c|c|c|c|c|c|}
\hline & $\begin{array}{l}\text { Total MM Patients } \\
(\mathrm{N}=64)\end{array}$ & $\begin{array}{l}\text { CPEO/KSS } \\
(\mathrm{N}=33)\end{array}$ & $\begin{array}{l}\text { MELAS/-like } \\
(\mathrm{N}=11)\end{array}$ & $\begin{array}{l}\text { MERRF } \\
(\mathrm{N}=3) \\
\end{array}$ & $\begin{array}{l}\text { Other MM } \\
(\mathrm{N}=17)\end{array}$ & $\begin{array}{l}\text { Controls } \\
(\mathrm{N}=25)\end{array}$ & $p$ value \\
\hline Age, yrs & $50 \pm 15$ & $52 \pm 14$ & $44 \pm 17$ & $46 \pm 3$ & $50 \pm 15$ & $47 \pm 12$ & 0.45 \\
\hline Male, n (\%) & $28(44)$ & $12(36)$ & $3(27)$ & $2(67)$ & $11(65)$ & $12(48)$ & 0.22 \\
\hline Diabetes, n (\%) & $10(16)$ & $5(15)$ & $3(27)$ & $0(0)$ & $2(12)$ & $3(12)$ & 0.51 \\
\hline Hypertension, n (\%) & $10(16)$ & $6(18)$ & $0(0)$ & $0(0)$ & $4(24)$ & $5(20)$ & 0.79 \\
\hline MM family history, n (\%) & $17(26)$ & $4(13)^{*}$ & $9(80)$ & $2(67)$ & $2(13)^{*}$ & - & $<0.001$ \\
\hline Previous cardiac diagnosis, n (\%) & $20(31)$ & $8(24)$ & $7(64)$ & $1(33)$ & $4(24)$ & - & 0.09 \\
\hline $\mathrm{BMl}, \mathrm{kg} / \mathrm{m}^{2}$ & $24 \pm 4$ & $23 \pm 4$ & $22 \pm 4$ & $25 \pm 3$ & $25 \pm 4$ & $26 \pm 4$ & 0.07 \\
\hline \multicolumn{8}{|l|}{ Lab results } \\
\hline Elevated CK, n (\%) & $29(45)$ & $14(42)$ & $5(46)$ & $2(67)$ & $8(47)$ & - & 0.89 \\
\hline Elevated TnT, n (\%) & $8(13)$ & $1(3)^{*}$ & $5(46)$ & $1(33)$ & $1(6)^{*}$ & - & 0.003 \\
\hline Elevated NT-proBNP, n (\%) & $16(25)$ & $10(30)$ & $4(36)$ & $0(0)$ & $2(11)$ & - & 0.29 \\
\hline Any elevated cardiac biomarkers, n (\%) & $21(33)$ & $11(33)$ & $6(55)$ & $1(33)$ & $3(18)$ & - & 0.23 \\
\hline \multicolumn{8}{|l|}{ ECG findings } \\
\hline Sinus rhythm, n (\%) & $64(100)$ & $33(100)$ & $11(100)$ & $3(100)$ & $17(100)$ & $25(100)$ & 1.00 \\
\hline QRS abnormalities, n (\%) & $11(17)$ & $5(15)$ & $5(46) \S$ & $1(33)$ & $0(0)^{*}$ & $2(8)$ & 0.01 \\
\hline ST/T abnormalities, n (\%) & $11(17)$ & $3(9)^{*}$ & $8(73) \S$ & $0(0)$ & $0(0)^{*}$ & $2(8)$ & $<0.001$ \\
\hline Any abnormal ECG findings, n (\%) & $18(28)$ & $8(24)^{*}$ & $9(82) \S$ & $1(33)^{*}$ & $0(0)^{*}$ & $4(16)$ & $<0.001$ \\
\hline \multicolumn{8}{|l|}{ Medication } \\
\hline ACE inhibitor/ARB, n (\%) & $16(25)$ & $9(27)$ & $3(20)$ & $0(0)$ & $5(29)$ & $5(20)$ & 0.89 \\
\hline Beta blocker, n (\%) & $9(14)$ & $5(15)$ & $1(10)$ & $0(0)$ & $3(18)$ & $2(8)$ & 0.90 \\
\hline CoQ10, n (\%) & $23(36)$ & $10(30)$ & $6(55)$ & $1(33)$ & $6(35)$ & - & 0.51 \\
\hline Creatin/Carnitin, n (\%) & $9(14)$ & $7(21)$ & $0(0)$ & $1(33)$ & $1(6)$ & - & 0.16 \\
\hline Vitamins, n (\%) & $15(24)$ & $9(27)$ & $0(0)$ & $1(33)$ & $5(31)$ & - & 0.15 \\
\hline
\end{tabular}

$\mathrm{MM}$ - mithochondrial myopathy; BMI - body mass index; CK - creatine kinase; TnT - troponin T; ACE - angiotensin converting enzyme; ARB - angiotensin receptor blocker; CoQ10 - coenzyme Q10

*- post Hoc $p<0.05$ vs. MELAS/-like; $\S$ - vs. Control

Bold data indicates either significant or most important results

Table 2 Frequency of clinical features

\begin{tabular}{|c|c|c|c|c|c|}
\hline $\mathrm{N}(\%)$ & Total MM Patients $(\mathrm{N}=64)$ & CPEO/KSS $(N=33)$ & MELAS/-like $(\mathrm{N}=11)$ & MERRF $(\mathrm{N}=3)$ & Other MM $(\mathrm{N}=17)$ \\
\hline Chest pain symptoms & $12(19)$ & $5(15)$ & $1(10)$ & $1(33)$ & $5(29)$ \\
\hline Exercise dyspnea & $30(47)$ & $17(52)$ & $2(18)$ & $3(100)$ & $8(47)$ \\
\hline Any cardiac symptom & $31(48)$ & $17(52)$ & $2(18)$ & $3(100)$ & $9(53)$ \\
\hline Skeletal myopathy & $44(69)$ & $23(70)$ & $9(82)$ & $2(67)$ & $10(59)$ \\
\hline Encephalopathy & $5(8)$ & $0(0)$ & $4(36)$ & $0(0)$ & $1(6)$ \\
\hline Seizures & $5(8)$ & $0(0)$ & $3(27)$ & $1(33)$ & $1(6)$ \\
\hline Stroke-like episodes & $5(8)$ & $0(0)$ & $4(36)$ & $0(0)$ & $1(6)$ \\
\hline Cognitive dysfunction & $5(8)$ & $1(3)$ & $3(27)$ & $0(0)$ & $1(6)$ \\
\hline Hearing loss & $10(16)$ & $5(15)$ & $4(36)$ & $0(0)$ & $1(6)$ \\
\hline Neuropathy & $11(16)$ & $3(9)$ & $1(9)$ & $1(33)$ & $6(35)$ \\
\hline Sleeping apnea & $3(5)$ & $1(3)$ & $0(0)$ & $1(33)$ & $1(6)$ \\
\hline Ptosis & $32(50)$ & $25(76)$ & $1(9)$ & $2(67)$ & $4(24)$ \\
\hline Ophtalmoplegia & $25(39)$ & $20(61)$ & $1(9)$ & 1 (33) & $3(18)$ \\
\hline Retinopathy & $6(9)$ & $3(9)$ & $2(18)$ & $0(0)$ & $1(6)$ \\
\hline
\end{tabular}


being found in 5 and NT-proBNP in 4. CPEO/ KSS and other MM patients showed significantly less frequently TnT elevation when compared to MELAS/-like $(\mathrm{p}=0.003)$. One third $(\mathrm{N}=21)$ of the MM patients had an increase in at least one of the two cardiac biomarkers with no significant difference between groups.

\section{ECG findings}

All patients and controls were in sinus rhythm at presentation. QRS abnormalities were seen in $11 \mathrm{MM}$ patients (left bundle branch block, $\mathrm{N}=1$; right bundle branch block, $\mathrm{N}=5$; pathologic $\mathrm{Q}$ waves, $\mathrm{N}=4$ and $\mathrm{LV}$ hypertrophy, $\mathrm{N}=1$ ) and in two controls (right bundle branch block, $\mathrm{N}=1$ and left ventricular hypertrophy, $\mathrm{N}=1$ ). Similarly, isolated $\mathrm{ST} / \mathrm{T}$ abnormalities were observed in $11 \mathrm{MM}$ patients (ST-segment depression, $\mathrm{N}=1$ and $\mathrm{T}$-wave inversion, $\mathrm{N}=10$ ) and in two controls (both with T-wave inversion). Five of the CPEO/KSS patients with abnormal ECG showed RBBB and 3 showed T-wave inversions. In the 9 (82\%) MELAS/-like patients with abnormal tracings, the following ECG abnormalities were found: pathologic Q-waves in four, LV hypertrophy in one, LBBB in one and ST/T changes in eight. In total, 18 (28 \%) MM patients showed abnormal ECG findings with significantly more MELAS/-like patients presenting with pathological ECGs compared to the other subgroups and to controls.

\section{CMR findings compared to normal controls}

The CMR findings for patients, including the different MM subgroups and controls are listed in Tables 3-4. Compared to controls, the total cohort of MM patients had significantly lower LV end-diastolic volumes $(p=0.028)$ and increased maximal wall thickness $(\mathrm{p}=0.005)$ with significant more frequent LV hypertrophy $(\mathrm{p}=0.016)$ and higher concentricity as expressed by the ratio of LV mass to end-diastolic volume $(\mathrm{p}<0.0001)$.

For the subgroup analysis, when compared to controls (Table 3), CPEO/KSS patients showed significantly lower LV end-diastolic volumes and increased LV concentricity without overt hypertrophy. On the other hand, in relation to controls, MELAS/-like patients presented with increased frequency of concentric hypertrophy with significantly larger wall thickness and LV mass to LV end-diastolic volume index. No significant differences regarding functional parameters and hypertrophy were depicted for MERRF and other MM when compared to controls.

Regarding LGE presence, 21 (33 \%) of the MM patients showed LGE in at least one myocardial segment, of which 19 (30\%) patients had non-ischemic patterns (16 intramural and two subepicardial) and two patients (both with CPEO) showed ischemic (subendocardial) LGE. Two patients with predominantly intramural LGE showed also limited transmural extension. Among controls, one patient showed ischemic and none non-ischemic LGE. Compared to controls, non-ischemic LGE was more frequently depicted in MM patients $(p=0.001)$. This significant difference was primarily due to the higher LGE prevalence in MELAS/-like $(\mathrm{N}=8,73 \%)$ and secondly in CPEO/KSS $(\mathrm{N}=10,30 \%)$ patients, but not in the other two subgroups (MERRF and other MM).

Altogether, 34 (53\%) MM patients presented at least one pathological CMR finding compared to only 3 (12\%)

Table 3 CMR findings (with controls)

\begin{tabular}{|c|c|c|c|c|c|c|c|}
\hline & $\begin{array}{l}\text { Total MM Patients } \\
(\mathrm{N}=64)\end{array}$ & $\begin{array}{l}\text { CPEO/KSS } \\
(\mathrm{N}=33)\end{array}$ & $\begin{array}{l}\text { MELAS/-like } \\
(N=11)\end{array}$ & $\begin{array}{l}\text { MERRF } \\
(\mathrm{N}=3)\end{array}$ & $\begin{array}{l}\text { Other MM } \\
(N=17)\end{array}$ & $\begin{array}{l}\text { Controls } \\
(\mathrm{N}=25)\end{array}$ & $p$ value \\
\hline LV end-diastolic volume index $\mathrm{ml} / \mathrm{m}^{2}$ & $67 \pm 18$ & $61 \pm 14^{*} \S$ & $85 \pm 24$ & $73 \pm 15$ & $66 \pm 14^{*}$ & $76 \pm 17$ & 0.001 \\
\hline LV end-systolic volume index, $\mathrm{ml} / \mathrm{m}^{2}$ & $25 \pm 2$ & $21 \pm 8^{*}$ & $37 \pm 20$ & $28 \pm 10$ & $24 \pm 7^{*}$ & $27 \pm 8$ & 0.002 \\
\hline LV mass index, $\mathrm{g} / \mathrm{m}^{2}$ & $56 \pm 23$ & $49 \pm 11^{*}$ & $90 \pm 35$ & $58 \pm 17$ & $50 \pm 12^{*}$ & $50 \pm 12^{*}$ & $<0.001$ \\
\hline LV ejection fraction, \% & $66 \pm 8$ & $66 \pm 7$ & $59 \pm 12$ & $62 \pm 5$ & $65 \pm 6$ & $65 \pm 5$ & 0.09 \\
\hline LV ejection fraction $<60 \%, \mathrm{n}(\%)$ & $18(28)$ & $8(24)$ & $6(55)$ & $1(33)$ & $3(18)$ & $2(8)$ & 0.07 \\
\hline LV mass/ end-diastolic volume, $\mathrm{g} / \mathrm{ml}$ & $0.84 \pm 0.27$ & $0.84 \pm 0.24 * \S$ & $1.07 \pm 0.31 \S$ & $0.71 \pm 0.06$ & $0.72 \pm 0.22^{*}$ & $0.67 \pm 0.11$ & $<0.001$ \\
\hline Max. wall thickness, mm & $10 \pm 3$ & $9 \pm 2^{*}$ & $14 \pm 3 \S$ & $10 \pm 2$ & $10 \pm 2^{*}$ & $8 \pm 2$ & $<0.001$ \\
\hline LV hypertrophy in absence of AHT, n (\%) & $14(22)$ & $3(9)^{*}$ & $9(82) \S$ & $1(33)$ & $1(6)^{*}$ & $0(0)^{*}$ & $<0.001$ \\
\hline RV end-diastolic volume index $\mathrm{ml} / \mathrm{m}^{2}$ & $66 \pm 14$ & $63 \pm 12$ & $69 \pm 18$ & $65 \pm 9$ & $72 \pm 15$ & $72 \pm 18$ & 0.21 \\
\hline $\mathrm{RV}$ end-systolic volume index, $\mathrm{ml} / \mathrm{m}^{2}$ & $30 \pm 9$ & $29 \pm 9$ & $30 \pm 10$ & $32 \pm 7$ & $33 \pm 10$ & $31 \pm 9$ & 0.49 \\
\hline RV ejection fraction, $\%$ & $55 \pm 10$ & $55 \pm 11$ & $57 \pm 8$ & $51 \pm 5$ & $54 \pm 10$ & $57 \pm 8$ & 0.70 \\
\hline LGE presence, n (\%) & $21(33)$ & $12(36) \S$ & $8(73) \S$ & $0(0)$ & $1(6)^{*}$ & $1(4)$ & $<0.001$ \\
\hline Non-ischemic LGE presence, n (\%) & $19(30)$ & $10(30) \S^{*}$ & $8(73) \S$ & $0(0)$ & $1(6)^{*}$ & $0(0)$ & $<0.001$ \\
\hline Any abnormal CMR findings, $\mathrm{n}(\%)$ & $34(53)$ & $18(55)$ & $10(91)$ & $1(33)$ & $5(29)$ & $3(12)$ & $<0.001$ \\
\hline
\end{tabular}

*- post Hoc $\mathrm{p}<0.05$ vs. MELAS/-like; $\S$ - vs. Control

LV - left ventricle; RV -right ventricle; AHT - arterial hypertension; LGE - late gadolinium enhancement; CMR - cardiac magnetic resonance Bold data indicates either significant or most important results 
Table 4 CMR findings (without controls)

\begin{tabular}{|c|c|c|c|c|c|c|}
\hline & Total $(\mathrm{N}=64)$ & CPEO/KSS $(\mathrm{N}=33)$ & MELAS/-like $(\mathrm{N}=11)$ & MERRF $(N=3)$ & Other MM $(\mathrm{N}=17)$ & $\mathrm{p}$ value \\
\hline LV end-diastolic volume index $\mathrm{ml} / \mathrm{m}^{2}$ & $67 \pm 18$ & $61 \pm 14^{*}$ & $85 \pm 24$ & $73 \pm 15$ & $66 \pm 14^{*}$ & 0.002 \\
\hline LV end-systolic volume index, $\mathrm{ml} / \mathrm{m}^{2}$ & $25 \pm 2$ & $21 \pm 8$ & $37 \pm 20$ & $28 \pm 10$ & $24 \pm 7$ & 0.003 \\
\hline LV mass index, $\mathrm{g} / \mathrm{m}^{2}$ & $56 \pm 23$ & $49 \pm 11^{*}$ & $90 \pm 35$ & $58 \pm 17$ & $50 \pm 12^{*}$ & $<0.001$ \\
\hline LV ejection fraction, \% & $66 \pm 8$ & $66 \pm 7$ & $59 \pm 12$ & $62 \pm 5$ & $65 \pm 6$ & 0.09 \\
\hline LV ejection fraction $<60 \%, \mathrm{n}(\%)$ & $18(28)$ & $8(24)$ & $6(55)$ & $1(33)$ & $3(18)$ & 0.26 \\
\hline LV mass/ end-diastolic volume, $\mathrm{g} / \mathrm{ml}$ & $0.84 \pm 0.27$ & $0.84 \pm 0.24$ & $1.07 \pm 0.31$ & $0.71 \pm 0.06$ & $0.72 \pm 0.22^{*}$ & 0.008 \\
\hline LV hypertrophy in absence of AHT, n (\%) & $14(22)$ & $3(9)^{*}$ & $9(82)$ & $1(33)$ & $1(6)^{*}$ & $<0.001$ \\
\hline RV end-diastolic volume index $\mathrm{ml} / \mathrm{m}^{2}$ & $66 \pm 14$ & $63 \pm 12$ & $69 \pm 18$ & $65 \pm 9$ & $72 \pm 15$ & 0.22 \\
\hline RV end-systolic volume index, $\mathrm{ml} / \mathrm{m}^{2}$ & $30 \pm 9$ & $29 \pm 9$ & $30 \pm 10$ & $32 \pm 7$ & $33 \pm 10$ & 0.39 \\
\hline RV ejection fraction, \% & $55 \pm 10$ & $55 \pm 11$ & $57 \pm 8$ & $51 \pm 5$ & $54 \pm 10$ & 0.75 \\
\hline LGE presence, n (\%) & $21(33)$ & $12(36)$ & $8(73)$ & $0(0)$ & $1(6)^{*}$ & 0.001 \\
\hline Non-ischemic LGE presence, n (\%) & $19(30)$ & $10(30)^{*}$ & $8(73)$ & $0(0)$ & $1(6)^{*}$ & $<0.001$ \\
\hline Any abnormal CMR findings, $\mathrm{n}(\%)$ & $34(53)$ & $18(55)^{*}$ & $10(91)$ & $1(33)$ & $5(29)^{*}$ & 0.007 \\
\hline
\end{tabular}

*- post Hoc $p<0.05$ vs. MELAS/-like

Bold data indicates either significant or most important results

control patients $(\mathrm{p}<0.0001)$. MELAS/-like patients had a pathological CMR in $91 \%(\mathrm{~N}=10)$ and $\mathrm{CPEO} / \mathrm{KSS}$ patients in $55 \%(\mathrm{~N}=18)$ of cases, respectively - which was significantly higher compared to the controls. As illustrated in Table 5, cardiac involvement was most frequently diagnosed by a comprehensive CMR study comprising cine- and LGE-images (in $53 \%$ ) and rather infrequently based on ECG or cardiac biomarkers, respectively (in $28 \%$ and $33 \%, \mathrm{p}=0.008)$.

\section{CMR pattern according to MM syndrome and relationship to other diagnostic tools CPEO/KSS}

A predominantly intramural pattern of enhancement was noted in 8/10 (80 \%) CPEO/KSS patients with proof of non-ischemic LGE (basal inferolateral distribution in six and basal to midventricular septal pattern in two; Fig. 1). A subepicardial pattern was found in the remaining two with basal inferolateral location in both. The three patients with LV hypertrophy in the absence of arterial hypertension or other causal reasons presented only isolated mild septal hypertrophy. There was no relationship between non- ischemic LGE presence or an abnormal CMR finding and an abnormal ECG ( $\mathrm{p}=0.67$ and $\mathrm{p}=0.23$, respectively). Moreover, there was no significant difference in the occurrence of elevated cardiac biomarkers between patients with $(\mathrm{N}=18)$ and without $(\mathrm{N}=15)$ abnormal CMR findings ( $29 \%$ vs. $38 \%, \mathrm{p}=0.72$ ) among $\mathrm{CPEO} / \mathrm{KSS}$ patients.

\section{MELAS/-like}

In comparison to CPEO/KSS, MELAS/-like patients showed significantly larger LV mass with increased LV end-diastolic volumes. Left ventricular hypertrophy was also more frequently encountered in this subgroup compared to CPEO/KSS. Particularly, a concentric pattern was noted in six out of nine $(67 \%)$ patients with $\mathrm{LV}$ hypertrophy. In addition, MELAS/-like patients showed higher rates for non-ischemic (predominantly intramural) LGE compared to CPEO/KSS. Moreover, a more heterogeneous distribution and extent of LGE, potentially appearing in any of the myocardial segments and occupying between one and 16 segments was observed in MELAS/-like patients (Fig. 2). Furthermore, a significant difference in the presence of an abnormal ECG

Table 5 Clinical vs. laboratory vs. imaging findings

\begin{tabular}{|c|c|c|c|c|c|c|c|}
\hline & $\begin{array}{l}\text { Total MM Patients } \\
(\mathrm{N}=64)\end{array}$ & $\begin{array}{l}\text { CPEO/KSS } \\
(\mathrm{N}=33)\end{array}$ & $\begin{array}{l}\text { MELAS/-like } \\
(N=11)\end{array}$ & $\begin{array}{l}\text { MERRF } \\
(N=3)\end{array}$ & $\begin{array}{l}\text { Other MM } \\
(\mathrm{N}=17)\end{array}$ & $\begin{array}{l}\text { Controls } \\
(N=25)\end{array}$ & $\mathrm{p}$ value \\
\hline Any (possibly) cardiac symptoms & $31(48)$ & $17(52)$ & $2(18)$ & $3(100)$ & $9(53)$ & - & \\
\hline Elevated TnT or NT-proBNP & $21(33)$ & $11(33)$ & $6(55)$ & $1(33)$ & $3(18)$ & - & 0.227 \\
\hline Any abnormal ECG findings & $18(28)$ & $8(24)^{*}$ & $9(82) \S$ & $1(33)^{*}$ & $0(0)^{*}$ & $2(8)$ & $<0.001$ \\
\hline LV ejection fraction $<60 \%, \mathrm{n}(\%)$ & $18(28)$ & $8(24)$ & $6(55)$ & $1(33)$ & $3(18)$ & $2(8)$ & 0.072 \\
\hline LGE presence, n (\%) & $21(33)$ & $12(36) \S$ & $8(73) \S$ & $0(0)$ & $1(6)^{*}$ & $1(6)$ & $<0.001$ \\
\hline Any abnormal CMR findings, n (\%) & $34(53)$ & $18(55) \S$ & $10(91) \S$ & $1(33)$ & $5(29)$ & $3(12)$ & $<0.001$ \\
\hline
\end{tabular}

*- post Hoc $p<0.05$ vs. MELAS/-like; $\S$ - vs. Control

Bold data indicates either significant or most important results 


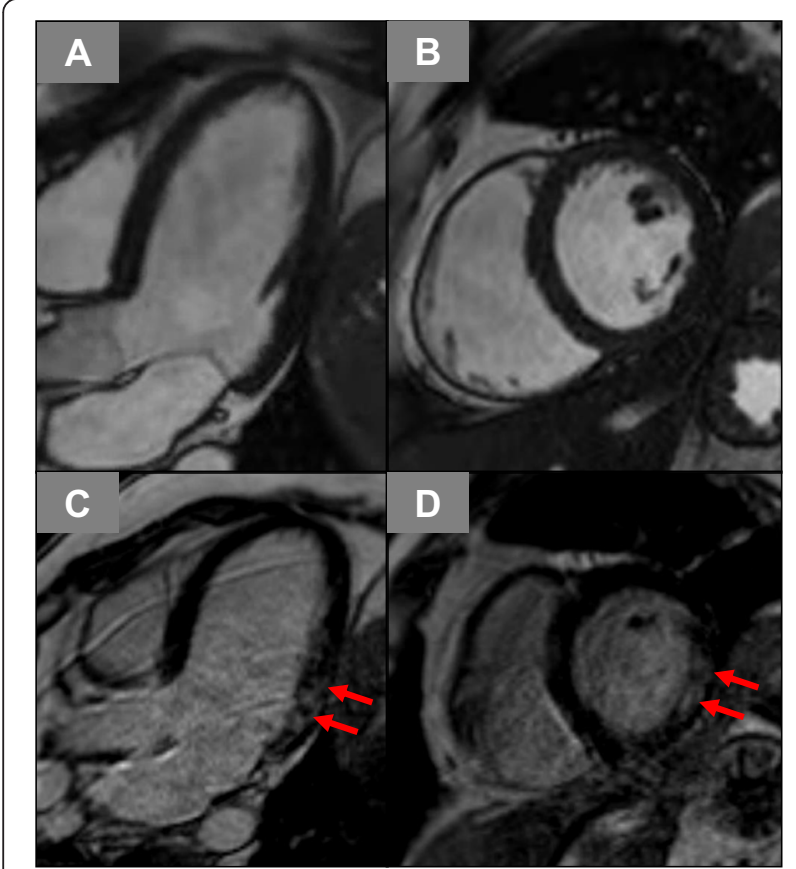

Fig. 1 Cine-CMR images in long-axis (a) and short-axis views (b) with corresponding late gadolinium enhancement (LGE) images (c-d). A diffuse intramural pattern of LGE is seen in the basal inferolateral wall segments of the left ventricle (red arrows) between patients with $(\mathrm{N}=8)$ and without $(\mathrm{N}=3)$ nonischemic LGE (100 \% vs. $33 \%, \mathrm{p}=0.050)$ could be noted. Moreover, the majority of patients (6/10) with abnormal CMR findings presented with elevated cardiac biomarkers; the only MELAS patient with a normal CMR showed no increase in biomarkers.

\section{MERRF}

Among the three MERRF patients only one showed pathological CMR findings with a mildly impaired LV ejection fraction and mild septal hypertrophy, however, without presence of LGE. The same patient presented a RBBB and mild TnT elevation.

\section{Other MM}

As shown in Table 3, among patients with other MM forms $(\mathrm{N}=17)$ only one showed presence of LGE (intramural pattern, location basal inferolateral) associated with mild septal hypertrophy. This LGE-positive patient also demonstrated a mild TnT elevation. Additionally, a mildly impaired LV ejection fraction was found in three patients, in two as an isolated finding and in one associated with mild septal hypertrophy. Another patient showed only isolated mild septal hypertrophy.

\section{Discussion}

Our study presents cross-sectional CMR data in a large cohort of adult patients with MM. We evaluated the presence and pattern of cardiomyopathy and could show

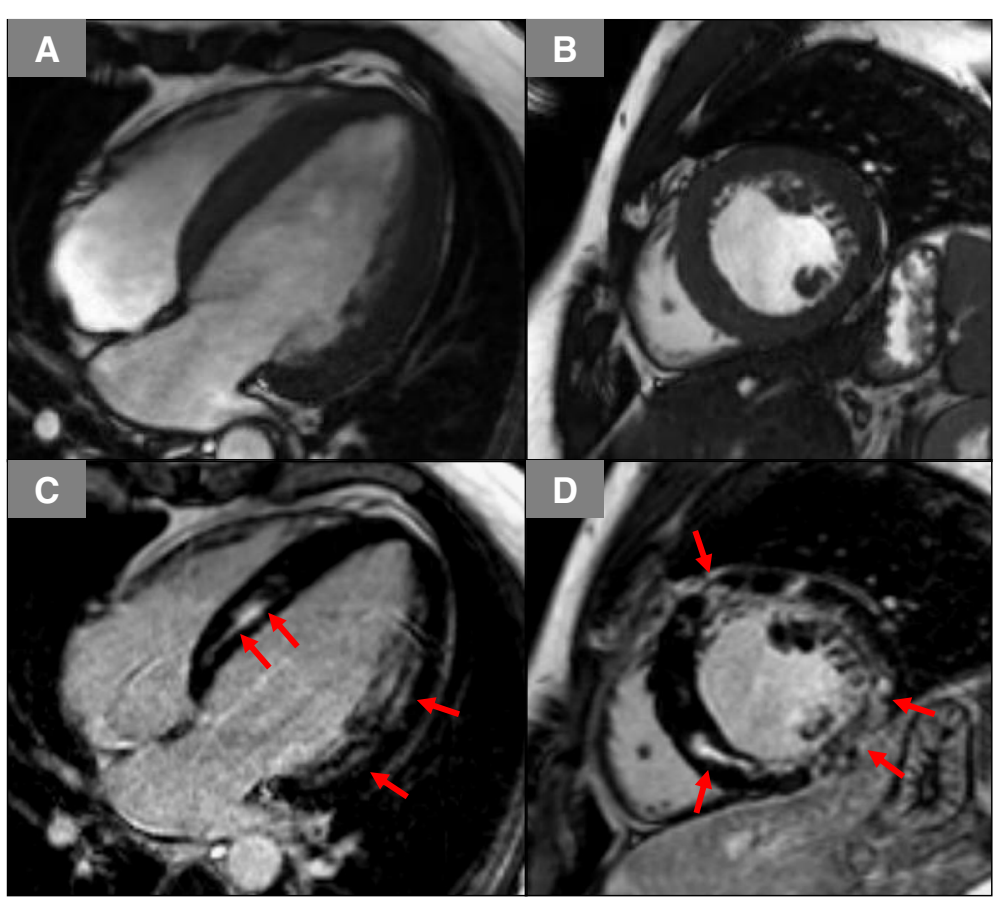

Fig. 2 Cine-CMR images in long-axis (a) and short-axis views (b) with corresponding late gadolinium enhancement (LGE) images (c-d). A diffuse, non-ischemic, patchy pattern of LGE is seen in almost all segments of the left ventricle (red arrows) 
that: (1) cardiac abnormalities are frequent among MM patients (53\%) with a non-ischemic LGE pattern being the most frequently encountered finding; (2) at least two different patterns of cardiac involvement can be distinguished, one in CPEO/KSS patients with intramural LGE in the basal inferolateral wall and a second one in MELAS/-like patients with overt concentric hypertrophy and intramural, diffusely located LGE, (3) in both CPEO/KSS and MELAS/-like patients CMR was superior in diagnosing cardiomyopathy compared to ECG and cardiac biomarkers, with a particular diagnostic benefit in CPEO/KSS patients.

\section{CMR abnormalities}

Even though MM-related cardiomyopathy was described in a series of reports, to the best of our knowledge the current work is the first to show that, by means of CMR, structural and/or functional abnormalities are present in the majority of MM patients [4, 6, 9, 10, 13, 14, 16-18]. Moreover, the present study is the first that consistently demonstrates the presence of a concentric hypertrophic remodelling pattern in MELAS/-like patients (and a tendency in CPEO/KSS patients) $[9,17]$. This remodelling pattern resembles the myocardial changes found in other inherited systemic disorders not associated with respiratory chain dysfunction such as Friedreich's ataxia [21-24]. In Friedreich's ataxia, such a remodelling pattern is generally considered to occur secondary to adaptive changes in response to an impaired cellular energy metabolism which is caused by mitochondrial structural abnormalities [7, 25, 26]. In MELAS, for example, histological examinations of endomyocardial biopsy specimens showed mitochondrial alterations with enlargement and accumulation and, secondarily, disperse interstitial fibrosis and partial myocardial disarray [7, 13, 15]. Considering that one third of MM patients presented with nonischemic LGE in the present study, CMR represents a new possibility to detect myocardial tissue damage in vivo beyond the known features of LV remodelling and functional abnormalities in this population [13-15, 18, 27].

\section{CMR-based phenotype patterns in different MM syndromes}

Whereas in the few patients with MERRF and in those with other MM no particular CMR changes were found, two potentially characteristic patterns were depicted in $\mathrm{CPEO} / \mathrm{KSS}$ and MELAS/-like patients, respectively. First, a tendency towards concentric remodelling was noted in $\mathrm{CPEO} / \mathrm{KSS}$ and second, an overt concentric hypertrophy in addition to LV systolic dysfunction (in half of the cases) was observed in MELAS/-like patients. At first view, these differences could be simply the consequence of a more severe cardiac involvement in MELAS/-like syndrome. Yet, interestingly, despite the known degree of neuromuscular phenotype overlap between syndromes, we found no overlap in cardiac pattern between the two syndromes [1]. Moreover, this difference becomes more prominent when looking at myocardial damage - as depicted by LGE-imaging.

As already reported previously, CPEO/KSS patients tend to show intramural LGE in the inferolateral wall that might either be due to an unequal and predominant distribution of mitochondrial energy deficiency in this area and/or due to an increased mechanical stress in the inferolateral wall [18]. In contrast, MELAS/-like patients do not only frequently show the presence of LGE, but the pattern of LGE is also completely different (Figs. 1-2): strong intramural LGE with focal (and rather patchy) accentuation extending in some cases over all myocardial segments. Besides replacement fibrosis secondary to perturbations in cell energy metabolism with consecutive cell death, another interesting mechanism might be involved in this unusual pattern of LGE seen in MELAS patients (Fig. 2): Similar to the pathogenesis of stroke like cerebral lesions, a hallmark of the MELAS syndrome, a mitochondrial angiopathy with consecutive vasogenic edema and fluid extravasation could be the mechanism for LGE formation in the myocardium $[11,13,16,28]$. This theory is further supported by the observed presence of myocardial edema as well as perfusion defects in areas with LGE in some of these patients - but not in LGE-positive areas from CPEO/KSS patients $[13,14]$.

Interestingly, despite the data suggesting a higher prevalence of arterial hypertension in MELAS as adjunctive cause of hypertrophy, none of our MELAS/-like patients had elevated blood pressure [29]. A clear explanation of the exact pathophysiology of these two patterns cannot be given and is beyond the purpose of this paper, but our findings demonstrate that despite the variability in phenotype within each of the two syndromes, relatively specific patterns of cardiomyopathy can be depicted for both.

\section{Additional value of CMR in the cardiac work-up}

First, CMR was superior to the ECG as well as to the measurement of cardiac biomarkers regarding the detection of (signs of) cardiomyopathy for both the whole study group and individual subgroups. Although ECG abnormalities are quite common and are sometimes seen early in the MM disease course, they are mostly unspecific [4, $8,30]$. For example, in a mixed MM population including CPEO and MELAS, Limongelli et al. described ECG changes in $68 \%$ of cases ranging from pre-excitation to conduction abnormalities and ST/T changes [4]. Similarly, in our MM population ECG abnormalities were multifaceted. Moreover, they did not relate to the functional and/ or structural CMR findings in CPEO/KSS and found only a weak relationship in MELAS/-like patients. Particularly in $\mathrm{CPEO} / \mathrm{KSS}$, approximately one third of the patients 
with abnormal CMR findings showed normal ECG and negative biomarkers.

Second, even though echocardiography was not routinely performed in the current study, we consider that CMR has a clear advantage over echocardiography for the diagnosis of cardiomyopathy in these patients: The superiority of CMR comes mainly from its ability to sensitively and reproducibly detect structural tissue changes with the depiction of subtle myocardial damage by LGEimaging $[18,31]$. Notably, in $15 \%$ of the CPEO/KSS patients LGE was the only pathological CMR finding. Further, as shown by Pfeffer et al. in a follow-up study conducted in CPEO patients with an initial normal cardiac work-up, only one of the fifteen patients developed new ECG and/or echocardiographic abnormalities over five years making the detection of cardiomyopathy by these techniques even less probable [10]. Therefore, we suggest that a CMR study should be part of the diagnostic approach of cardiomyopathy in MM patients. Early detection of cardiac disease is a prerequisite for the implementation of successful therapeutic strategies and appropriate risk stratification with timing of follow-up studies. In this context, the recently published ESC guidelines for the management of hypertrophic cardiomyopathy also address mitochondrial diseases such as MELAS and suggest to perform a CMR study at initial presentation if local expertise in this technique is available [32]. We advocate that CMR should be considered in all patients with $\mathrm{MM}$ at their baseline assessment - if local resources and expertise permit it. Moreover, we suggest that CMR follow-up studies in MM patients with pathological CMR results (particularly with presence of LGE and/or impaired systolic function) and potentially progressive disease particularly those with MELAS and MELAS-like disease should be considered every 6-12 months while a follow-up study every 4-5 years will be sufficient in (adult) patients with no involvement of the heart muscle.

In MM patients with a rather hypertrophic pattern of cardiomyopathy (e.g. MELAS or MELAS-like patients), ß-blockers, angiotensin-converting enzyme (ACE) inhibitors and angiotensin receptor blockers may potentially help to (at least) slow down the progression of cardiomyopathy - as was shown in aetiologically different forms of HCM [32]. In this context, it should be emphasized that MM such as MELAS and MELAS-like diseases are mentioned as specific HCM forms in the recently published "2014 ESC guidelines on diagnosis and management of HCM".

CMR may not only help to accurately assess the thickness of the LV wall, but the mere presence of LGE in e.g. MELAS patients may precede the occurrence of LV hypertrophy and thereby used as a diagnostic tool for a timely initiation of the aforementioned treatment options - prior to the occurrence of LV hypertrophy and advanced myocardial scarring. Our own (unpublished preliminary) experiences suggest that cardiac disease progression regarding myocardial scarring occurs surprisingly fast in some patients with MELAS or MELAS-like diseases. Therefore, the most accurate diagnostic tools available should be used in order to detect the first signs of cardiomyopathy.

Paralleling other genetic cardiomyopathies, e.g. hypertrophic cardiomyopathy, non-ischemic LGE detection could play an additional prognostic role in the risk stratification of MM, particularly in MELAS patients [33-35]. It has been already shown that MELAS patients have a high incidence of cardiac death as well as arrhythmia and heart failure events, primarily related to LV hypertrophy $[2,3]$. Since our preliminary experiences in MELAS/-like patients point to a rather disproportionately extensive pattern of LGE compared to the degree of LV hypertrophy (Fig. 1), one may even hypothesize that detection of LGE in these patients could have a superior prognostic value than in other non-MM diseases that are associated with LV hypertrophy. However, this issue is a clinically relevant topic for future research and so far, there are no follow-up data involving LGE-CMR in MM patients.

\section{Limitations}

The first limitation is the small number of patients, particularly suffering from MERRF. This fact obviously limits us to draw appropriate conclusions regarding presence, pattern and clinical value of cardiomyopathy in these patients. A recent study from Wahbi et al. found cardiac abnormalities in $44 \%$ of the 18 MERRF patients included comprising LV dilatation or hypertrophy with or without systolic dysfunction [12]. A direct comparison to our data is difficult as this study used echocardiography and other definitions for LV hypertrophy and dilatation.

A second limitation is the absence of histopathological data. The patients did not fulfil the current guideline recommendations for performing EMB and therefore, this procedure was rarely performed [36].

A third limitation lies in the fact that controls were free from cardiac symptoms while MM patients were symptomatic at inclusion. Nevertheless, in MM patients cardiac symptoms are often unspecific and rather difficult to differentiate from the coexisting neuromuscular disease. Moreover, no association between symptoms and CMR findings was found.

\section{Conclusion}

Cardiac involvement is a frequent finding in MM patients - and particularly present in KSS/CPEO as well as MELAS/-like patients. Despite a high variability in clinical presentation, CPEO/KSS patients typically show an intramural pattern of LGE in the basal inferolateral wall whereas MELAS patients are characterized by overt concentric hypertrophy and a rather unique, focally accentuated and diffusely distributed LGE. 


\section{Additional file}

Additional file 1: Table S1. Detailed overview of underlying genetic mutations.

\section{Abbreviations}

BNP: Brain natriuretic-peptide; CK: Creatine kinase; CMR: Cardiovascular magnetic resonance; CPEO: Chronic progressive external ophthalmoplegia; DCM: Dilative cardiomyopathy; LGE: Late-gadolinium-enhāncement; LV: Left ventricle; LV-EDV: Left ventricular end-diastolic volume; LV-ESV: Left ventricular end-systolic volume; LV-EF: Left ventricular ejection fraction; KSS: Kearns-Sayre syndrome; MELAS: Mitochondrial encephalopathy with lactic acidosis and stroke-like episodes; MERRF: Myoclonic epilepsy with ragge $\bar{d}$-red fibers; $\overline{\mathrm{M} M}$ : Mitochondrial myopathy; RV: Right ventricle; RV-EDV: 'Right ventricular end-diastolic volume; SSFP: Steady-state-free-precession; TnT: Troponin T.

\section{Competing interests}

The authors declare that they have no competing interests.

\section{Authors' contributions}

AF participated in the CMR exams, carried out the data and statistical analysis, and wrote the initial draft version of the manuscript. AL participated in the CMR exams and in the analysis of the CMR data. BSD, MB and PJ were involved in the recruitment of study patients and critically reviewed the manuscript. JW was involved in the recruitment of study patients. SR participated in the CMR exams and in the analysis of the CMR data. US provided additional supervision and critically reviewed the manuscript. AY supervised the study, critically reviewed the manuscript and drafted the manuscript. All authors read and approved the final manuscript.

\section{Acknowledgements}

None.

\section{Funding sources}

This work was financially supported by a grant from the Robert-Bosch-Stiftung (grant to A.Y. and U.S.) and a grant by the Deutsche Gesellschaft für Muskelkranke (Yi1/1 to A.Y).

\section{Author details}

'Department of Cardiology and Angiology, University Hospital Münster, Albert-Schweitzer-Campus 1, building A1, 48149 Münster, Germany. ${ }^{2}$ Division of Cardiology, Robert-Bosch-Krankenhaus, Stuttgart, Germany. ${ }^{3}$ Department of Sleep Medicine and Neuromuscular Disorders, University Hospital Münster, Münster, Germany.

\section{Received: 8 December 2014 Accepted: 6 May 2015}

\section{Published online: 22 May 2015}

\section{References}

1. Pfeffer G, Chinnery PF. Diagnosis and treatment of mitochondrial myopathies. Ann Med. 2013;45:4-16.

2. Holmgren D, Wahlander H, Eriksson BO, Oldfors A, Holme E, Tulinius M. Cardiomyopathy in children with mitochondrial disease; clinical course and cardiological findings. Eur Heart J. 2003;24:280-8

3. Klopstock T, Jaksch M, Gasser T. Age and cause of death in mitochondrial diseases. Neurology. 1999;53:855-7.

4. Limongelli G, Tome-Esteban M, Dejthevaporn C, Rahman S, Hanna MG, Elliott PM. Prevalence and natural history of heart disease in adults with primary mitochondrial respiratory chain disease. Eur J Heart Fail. 2010:12:114-21.

5. Scaglia F, Towbin JA, Craigen WJ, Belmont JW, Smith EO, Neish SR, et al. Clinical spectrum, morbidity, and mortality in 113 pediatric patients with mitochondrial disease. Pediatrics. 2004;114:925-31.

6. Bates MG, Bourke JP, Giordano C, d'Amati G, Turnbull DM, Taylor RW. Cardiac involvement in mitochondrial DNA disease: clinical spectrum, diagnosis, and management. Eur Heart J. 2012:33:3023-33.

7. Meyers DE, Basha HI, Koenig MK. Mitochondrial cardiomyopathy: pathophysiology, diagnosis, and management. Tex Heart Inst J. 2013:40:385-94.
8. Baik R, Chae JH, Lee YM, Kang HC, Lee JS, Kim HD. Electrocardiography as an early cardiac screening test in children with mitochondrial disease. Korean J Pediatr. 2010;53:644-7

9. Majamaa-Voltti K, Peuhkurinen K, Kortelainen ML, Hassinen IE, Majamaa K. Cardiac abnormalities in patients with mitochondrial DNA mutation 3243A > G. BMC Cardiovasc Disord. 2002;2:12.

10. Pfeffer G, Mezei MM. Cardiac screening investigations in adult-onset progressive external ophthalmoplegia patients. Muscle Nerve. 2012:46:593-6.

11. Sato W, Tanaka M, Sugiyama S, Nemoto T, Harada K, Miura Y, et al. Cardiomyopathy and angiopathy in patients with mitochondrial myopathy, encephalopathy, lactic acidosis, and strokelike episodes. Am Heart J. 1994;128:733-41.

12. Wahbi K, Larue S, Jardel C, Meune C, Stojkovic T, Ziegler F, et al. Cardiac involvement is frequent in patients with the m.8344A $>\mathrm{G}$ mutation of mitochondrial DNA. Neurology. 2010;74:674-7.

13. Jose T, Gdynia HJ, Mahrholdt H, Vohringer M, Klingel K, Kandolf R, et al. CMR gives clue to "ragged red fibers" in the heart in a patient with mitochondrial myopathy. Int J Cardiol. 2011;149:e24-7.

14. Nakanishi M, Harada M, Tadamura E, Kotani H, Kawakami R, Kuwahara K, et al. Images in cardiovascular medicine. Mitochondrial cardiomyopathy evaluated with cardiac magnetic resonance. Circulation. 2007:116:e25-6.

15. Palecek T, Tesarova M, Kuchynka P, Dytrych V, Elleder M, Hulkova H, et al. Hypertrophic cardiomyopathy due to the mitochondrial DNA mutation m.3303C > T diagnosed in an adult male. Int Heart J. 2012;53:383-7.

16. Partington SL, Givertz MM, Gupta S, Kwong RY. Cardiac magnetic resonance aids in the diagnosis of mitochondrial cardiomyopathy. Circulation. 2011;123:e227-9.

17. Bates MG, Hollingsworth KG, Newman JH, Jakovljevic DG, Blamire AM, MacGowan GA, et al. Concentric hypertrophic remodelling and subendocardial dysfunction in mitochondrial DNA point mutation carriers. Eur Heart J Cardiovasc Imaging. 2013;14:650-8

18. Yilmaz A, Gdynia HJ, Ponfick M, Rosch S, Lindner A, Ludolph AC, et al. Cardiovascular magnetic resonance imaging (CMR) reveals characteristic pattern of myocardial damage in patients with mitochondrial myopathy. Clin Res Cardiol. 2012;101:255-61.

19. McMurray JJ, Adamopoulos S, Anker SD, Auricchio A, Bohm M, Dickstein K et al. ESC guidelines for the diagnosis and treatment of acute and chronic heart failure 2012: The Task Force for the Diagnosis and Treatment of Acute and Chronic Heart Failure 2012 of the European Society of Cardiology. Developed in collaboration with the Heart Failure Association (HFA) of the ESC. Eur J Heart Fail. 2012;14:803-69.

20. Khouri MG, Peshock RM, Ayers CR, de Lemos JA, Drazner MH. A 4-tiered classification of left ventricular hypertrophy based on left ventricular geometry: the Dallas heart study. Circ Cardiovasc Imaging. 2010;3:164-71.

21. Cheng S, Fernandes VR, Bluemke DA, McClelland RL, Kronmal RA, Lima JA Age-related left ventricular remodeling and associated risk for cardiovascular outcomes: the Multi-Ethnic Study of Atherosclerosis. Circ Cardiovasc Imaging. 2009;2:191-8.

22. Dutta D, Calvani R, Bernabei R, Leeuwenburgh C, Marzetti E. Contribution of impaired mitochondrial autophagy to cardiac aging: mechanisms and therapeutic opportunities. Circ Res. 2012;110:1125-38.

23. Raman SV, Phatak K, Hoyle JC, Pennell ML, McCarthy B, Tran T, et al. Impaired myocardial perfusion reserve and fibrosis in Friedreich ataxia: a mitochondrial cardiomyopathy with metabolic syndrome. Eur Heart J. 2011;32:561-7.

24. Weidemann F, Stork S, Liu D, Hu K, Herrmann S, Ertl G, et al. Cardiomyopathy of Friedreich ataxia. J Neurochem. 2013;126 Suppl 1:88-93.

25. DiMauro S, Schon EA. Mitochondrial respiratory-chain diseases. N Engl J Med. 2003;348:2656-68.

26. Dominic EA, Ramezani A, Anker SD, Verma M, Mehta N, Rao M. Mitochondrial cytopathies and cardiovascular disease. Heart. 2014;100:611-8.

27. Stalder N, Yarol N, Tozzi P, Rotman S, Morris M, Fellmann F, et al. Mitochondrial A3243G mutation with manifestation of acute dilated cardiomyopathy. Circ Heart Fail. 2012:5:e1-3.

28. Finsterer J. MELAS in the heart. Int J Cardiol. 2009;137:e65-6.

29. Hannah-Shmouni F, Sirrs S, Mezei MM, Waters PJ, Mattman A. Increased prevalence of hypertension in young adults with high heteroplasmy levels of the MELAS m.3243A > G mutation. JIMD Rep. 2014;12:17-23.

30. Finsterer J, Stöllberger C, Blazek G, Kunafer M, Prager E. Cardiac involvement over 10 years in myotonic and Becker muscular dystrophy and mitochondrial disorder. Int J Cardiol. 2007;119(2):176-84. 
31. Myerson SG, Montgomery HE, Whittingham M, Jubb M, World MJ, Humphries SE, et al. Left ventricular hypertrophy with exercise and ACE gene insertion/deletion polymorphism: a randomized controlled trial with losartan. Circulation. 2001;103:226-30.

32. Elliott PM, Anastasakis A, Borger MA, Borggrefe M, Cecchi F, Charron P, et al. 2014 ESC Guidelines on diagnosis and management of hypertrophic cardiomyopathy: The task force for the diagnosis and management of hypertrophic cardiomyopathy of the European Society of Cardiology (ESC). Eur Heart J. 2014;35:2733-79.

33. Bruder O, Wagner A, Jensen CJ, Schneider S, Ong P, Kispert EM, et al. Myocardial scar visualized by cardiovascular magnetic resonance imaging predicts major adverse events in patients with hypertrophic cardiomyopathy. J Am Coll Cardiol. 2010;56:875-87.

34. Ismail TF, Jabbour A, Gulati A, Mallorie A, Raza S, Cowling TE, et al. Role of late gadolinium enhancement cardiovascular magnetic resonance in the risk stratification of hypertrophic cardiomyopathy. Heart. 2014;100(23):1851-8.

35. O'Hanlon R, Grasso A, Roughton M, Moon JC, Clark S, Wage R, et al. Prognostic significance of myocardial fibrosis in hypertrophic cardiomyopathy. J Am Coll Cardiol. 2010;56:867-74.

36. Cooper LT, Baughman KL, Feldman AM, Frustaci A, Jessup M, Kuhl U, et al. The role of endomyocardial biopsy in the management of cardiovascular disease: a scientific statement from the American Heart Association, the American College of Cardiology, and the European Society of Cardiology. Endorsed by the Heart Failure Society of America and the Heart Failure Association of the European Society of Cardiology. J Am Coll Cardiol. 2007;50:1914-31.

\section{Submit your next manuscript to BioMed Central and take full advantage of:}

- Convenient online submission

- Thorough peer review

- No space constraints or color figure charges

- Immediate publication on acceptance

- Inclusion in PubMed, CAS, Scopus and Google Scholar

- Research which is freely available for redistribution 